\title{
WEIGHTED HARDY-TYPE INEQUALITIES INVOLVING FRACTIONAL CALCULUS OPERATORS
}

\author{
Sajid Iqbal, Josip PeČarić, Muhammad Samraiz and Živorad \\ TOMOVSKI
}

\begin{abstract}
The aim of this paper is to give a new class of general weighted Hardy-type inequalities involving an arbitrary convex function with some applications of generalized fractional calculus convolutive operators which contain Gauss-hypergeometric function, generalized MittagLeffler function and Hilfer fractional derivative operator, in the kernel
\end{abstract}

\section{INTRODUCTION}

In recent years many mathematicians gave generalizations and improvements of Hardy-type inequalities. They discover important and useful Hardytype integral inequalities for convex functions which has great importance in mathematical analysis. The Hardy inequality has fundamental importance in the mathematical analysis and lot of rich literature and informations concerning Hardy-type inequalities have been published (see for example $[1-3,5,11-13])$.

Let $\left(\Omega_{1}, \Sigma_{1}, \mu_{1}\right)$ and $\left(\Omega_{2}, \Sigma_{2}, \mu_{2}\right)$ be measure spaces with $\sigma$-finite measures and $A_{k}$ be an integral operator defined by

$$
A_{k} f(x):=\frac{1}{K(x)} \int_{\Omega_{2}} k(x, y) f(y) d \mu_{2}(y),
$$

where $k: \Omega_{1} \times \Omega_{2} \rightarrow \mathbb{R}$ is measurable and non-negative kernel, $f$ is measurable function on $\Omega_{2}$, and

$$
0<K(x):=\int_{\Omega_{2}} k(x, y) d \mu_{2}(y), \quad x \in \Omega_{1} .
$$

The following results of this section are given in [3].

2010 Mathematics Subject Classification. 26D15, 26D10, 26A33, 34B27.

Key words and phrases. Inequalities, convex function, fractional derivatives, generalized fractional integral operator. 
TheOREM 1.1. Let $0<p \leq q<\infty$. Let $\left(\Omega_{1}, \Sigma_{1}, \mu_{1}\right)$ and $\left(\Omega_{2}, \Sigma_{2}, \mu_{2}\right)$ be measure spaces with $\sigma$-finite measures, $u$ be a weight function on $\Omega_{1}$, w be a $\mu_{2}-$ a.e. positive function on $\Omega_{2}, k$ be a non-negative measurable function on $\Omega_{1} \times \Omega_{2}$, and $K$ be defined on $\Omega_{1}$ by (1.2). Suppose that $K(x)>0$ for all $x \in \Omega_{1}$ and that the function $x \mapsto u(x)\left(\frac{k(x, y)}{K(x)}\right)^{\frac{q}{p}}$ is integrable on $\Omega_{1}$ for each fixed $y \in \Omega_{2}$. Let $\Phi$ be a non-negative convex function on an interval $I \subseteq \mathbb{R}$. If

$$
A=\sup _{y \in \Omega_{2}} w^{\frac{-1}{p}}(y)\left(\int_{\Omega_{1}} u(x)\left(\frac{k(x, y)}{K(x)}\right)^{\frac{q}{p}} d \mu_{1}(x)\right)^{\frac{1}{q}}<\infty,
$$

then there exists a positive constant $C$, such that the inequality

$$
\left(\int_{\Omega_{1}} u(x)\left[\Phi\left(A_{k} f(x)\right)\right]^{\frac{q}{p}} d \mu_{1}(x)\right)^{\frac{1}{q}} \leq C\left(\int_{\Omega_{2}} w(y) \Phi(f(y)) d \mu_{2}(y)\right)^{\frac{1}{p}}
$$

holds for all measurable functions $f: \Omega_{2} \rightarrow \mathbb{R}$ with values in $I$ and $A_{k} f$ be defined by (1.1). Moreover, if $C$ is smallest constant for (1.3) to hold, then $C \leq A$.

Corollary 1.2. Let $-\infty<q \leq p<0$, and let the assumption of Theorem 1.1 be satisfied with a positive convex function $\Phi$. If

$$
B=\inf _{y \in \Omega_{2}} w^{\frac{-1}{p}}(y)\left(\int_{\Omega_{1}} u(x)\left(\frac{k(x, y)}{K(x)}\right)^{\frac{q}{p}} d \mu_{1}(x)\right)^{\frac{1}{q}}<\infty,
$$

then there exists a positive real constant $C$, such that the inequality

$$
\begin{aligned}
& \left(\int_{\Omega_{1}} u(x)\left[\Phi\left(A_{k} f(x)\right)\right]^{\frac{q}{p}} d \mu_{1}(x)\right)^{\frac{1}{q}} \\
& \geq C\left(\int_{\Omega_{2}} w(y) \Phi(f(y)) d \mu_{2}(y)\right)^{\frac{1}{p}}
\end{aligned}
$$

holds for all measurable functions $f: \Omega_{2} \rightarrow \mathbb{R}$ with values in $\Omega_{2}$. Moreover, if $C$ is smallest constant for (1.4) to hold, then $C \geq B$.

Theorem 1.3. Let $1<p \leq q<\infty$. Let $\left(\Omega_{1}, \Sigma_{1}, \mu_{1}\right)$ and $\left(\Omega_{2}, \Sigma_{2}, \mu_{2}\right)$ be measure spaces with $\sigma$-finite measures, $u$ be a weight function on $\Omega_{1}$, $v$ be a measurable $\mu_{2}$-a.e. positive function on $\Omega_{2}, k$ be a non-negative measurable function on $\Omega_{1} \times \Omega_{2}$, and $K$ be defined on $\Omega_{1}$ by (1.2). Let $K(x)>0$ for all $x \in \Omega_{1}$ and let the function $x \mapsto u(x)\left(\frac{k(x, y)}{K(x)}\right)^{q}$ be integrable on $\Omega_{1}$ for each 
fixed $y \in \Omega_{2}$. Suppose that $\Phi: I \rightarrow[0, \infty)$ is a bijective convex function on an interval $I \subseteq \mathbb{R}$. If there exist a real parameter $s \in(1, p)$ and a positive measurable function $V: \Omega_{2} \rightarrow \mathbb{R}$ such that

$$
A(s, V)=F(V, v) \sup _{y \in \Omega_{2}} V^{\frac{s-1}{p}}(y)\left(\int_{\Omega_{1}} u(x)\left(\frac{k(x, y)}{K(x)}\right)^{q} d \mu_{1}(x)\right)^{\frac{1}{q}}<\infty,
$$

where

$$
F(V, v)=\left(\int_{\Omega_{2}} V^{\frac{-p^{\prime}(s-1)}{p}}(y) v^{1-p^{\prime}}(y) d \mu_{2}(y)\right)^{\frac{1}{p^{\prime}}},
$$

then there is a positive real constant $C$, such that the inequality

$$
\left(\int_{\Omega_{1}} u(x) \Phi^{q}\left(A_{k} f(x)\right) d \mu_{1}(x)\right)^{\frac{1}{q}} \leq C\left(\int_{\Omega_{2}} v(y) \Phi^{p}(f(y)) d \mu_{2}(y)\right)^{\frac{1}{p}}
$$

holds for all measurable functions $f: \Omega_{2} \rightarrow \mathbb{R}$ with values in $I$ where $A_{k} f$ is defined on $\Omega_{1}$ by (1.1). Moreover, if $C$ is smallest constant for (1.5) to hold, then

$$
C \leq \inf _{\substack{1<s<p \\ V>0}} A(s, V)
$$

\section{HARDY-TyPe INEQUALITIES FOR FRACTIONAL INTEGRAL OPERATOR} WHICH CONTAINS GAUSS-HYPERGEOMETRIC FUNCTION

First we give the definition of fractional hypergeometric operator (see [4]).

Definition 2.1. Let $\alpha>0, \mu>-1, \beta, \eta \in \mathbb{R}$. Then the generalized fractional integral $I_{a, t}^{\alpha, \beta, \eta, \mu}$ of order $\alpha$, for a real-valued continuous function $f$ is defined by:

$$
\begin{aligned}
& \text { (2.1) } I_{0, x}^{\alpha, \beta, \eta, \mu} f(x) \\
& =\frac{x^{-\alpha-\beta-2 \mu}}{\Gamma(\alpha)} \int_{0}^{x} t^{\mu}(x-t)^{\alpha-1}{ }_{2} F_{1}\left(\alpha+\beta+\mu,-\eta ; \alpha ; 1-\frac{t}{x}\right) f(t) d t, x \in[0, b],
\end{aligned}
$$

where, the function ${ }_{2} F_{1}(., ., ;$.$) appearing as kernel for operator (2.1) is the$ Gaussian hypergeometric function defined by

$$
{ }_{2} F_{1}(a, b ; c ; t)=\sum_{n=0}^{\infty} \frac{(a)_{n}(b)_{n}}{(c)_{n} n !} t^{n},
$$

and $(a)_{n}$ is the Pochhammer symbol: $(a)_{n}=a(a+1) \ldots(a+n-1),(a)_{0}=1$. 
The operator (2.1) includes Saigo, Riemann-Liouville and Erdélyi-Kober fractional integral operators i.e.

$$
\begin{aligned}
& I_{0, x}^{\alpha, \beta, \eta} f(x)=I_{0, x}^{\alpha, \beta, \eta, 0} f(x) \\
&=\frac{x^{-\alpha-\beta}}{\Gamma(\alpha)} \int_{0}^{x}(x-t)^{\alpha-1}{ }_{2} F_{1}\left(\alpha+\beta,-\eta ; \alpha ; 1-\frac{t}{x}\right) f(t) d t, x \in[0, b] . \\
& R^{\alpha} f(x)=I_{0, x}^{\alpha,-\alpha, \eta} f(x)=\frac{1}{\Gamma(\alpha)} \int_{0}^{x}(x-t)^{\alpha-1} f(t) d t, x \in[0, b] .
\end{aligned}
$$

and

$$
I^{\alpha, \eta} f(x)=I_{0, x}^{\alpha, 0, \eta} f(x)=\frac{x^{-\alpha-\eta}}{\Gamma(\alpha)} \int_{0}^{x}(x-t)^{\alpha-1} t^{\eta} f(t) d t, x \in[0, b] .
$$

Our first result for generalized fractional integral operator is given in the upcoming theorem.

TheOREM 2.2. Let $0<p \leq q<\infty, s>1, \alpha>0, \mu>-1, \beta, \eta \in \mathbb{R}$. Let $u$ be a weight function on $(0, b), w$ be a.e. positive function on $(0, b)$. If

(2.2) $A=\sup _{t \in(0, b)} w^{\frac{-1}{p}}(t)\left(\frac{\Gamma(1-\beta) \Gamma(\alpha+\mu+1+\eta)}{\Gamma(\mu+1) \Gamma(1-\beta+\eta)}\right)^{\frac{1}{p}}$

$\times\left(\int_{t}^{b} u(x)\left(x^{-\alpha-\mu}{ }_{2} F_{1}\left(\alpha+\beta+\mu,-\eta, \alpha ; 1-\frac{t}{x}\right) t^{\mu}(x-t)^{\alpha-1}\right)^{\frac{q}{p}} d x\right)^{\frac{1}{q}}<\infty$.

Then there exists a positive constant $C$, such that the inequality

$$
\begin{gathered}
\left(\int_{0}^{b} u(x)\left(\frac{I_{0, x}^{\alpha, \beta, \eta, \mu} f(x) \Gamma(1-\beta) \Gamma(\alpha+\mu+1+\eta)}{x^{-\mu-\beta} \Gamma(\mu+1) \Gamma(1-\beta+\eta)}\right)^{\frac{s q}{p}} d x\right)^{\frac{1}{q}} \\
\leq C\left(\int_{0}^{b} w(t) f^{s}(t) d t\right)^{\frac{1}{p}}
\end{gathered}
$$

holds for all measurable functions $f: I \rightarrow \mathbb{R}$ with values in $I$. Moreover, if $C$ is the smallest constant for (2.3) to hold, then $C \leq A$.

Proof. Applying Theorem 1.1 with $\Omega_{1}=\Omega_{2}=(0, b), d \mu_{1}(x)=d x$, $d \mu_{2}(t)=d t$,

$$
\hat{k}(x, t)= \begin{cases}\frac{x^{-\alpha-\beta-2 \mu}}{\Gamma(\alpha)}{ }_{2} F_{1}\left(\alpha+\beta+\mu,-\eta, \alpha ; 1-\frac{t}{x}\right) t^{\mu}(x-t)^{\alpha-1}, & 0 \leq t \leq x \\ 0, & x<t \leq b\end{cases}
$$


and we calculate $\hat{K}(x)$ as:

(2.4) $\hat{K}(x)=\frac{1}{\Gamma(\alpha)} \int_{0}^{x} x^{-\alpha-\beta-2 \mu_{2}} F_{1}\left(\alpha+\beta+\mu,-\eta, \alpha ; 1-\frac{t}{x}\right) t^{\mu}(x-t)^{\alpha-1} d t$

Substituting $1-\frac{t}{x}=y$ and using the formula given in [6, page 813], i.e.

$$
\int_{0}^{1} x^{\gamma-1}(1-x)^{\rho-1} F(\alpha, \beta ; \gamma ; x) d x=\frac{\Gamma(\gamma) \Gamma(\rho) \Gamma(\gamma+\rho-\alpha-\beta)}{\Gamma(\gamma+\rho-\alpha) \Gamma(\gamma+\rho-\beta)},
$$

we get

$$
\hat{K}(x)=\frac{x^{-\mu-\beta} \Gamma(\mu+1) \Gamma(1-\beta+\eta)}{\Gamma(1-\beta) \Gamma(\alpha+\mu+1+\eta)} .
$$

So that $A_{k} f(x)$ becomes

$$
A_{k} f(x)=\frac{I_{0, x}^{\alpha, \beta, \eta, \mu} f(x) \Gamma(1-\beta) \Gamma(\alpha+\mu+1+\eta)}{x^{-\mu-\beta} \Gamma(\mu+1) \Gamma(1-\beta+\eta)} .
$$

Then the inequality (1.3) takes the form

$$
\begin{gathered}
\left(\int_{0}^{b} u(x)\left[\Phi\left(\frac{I_{0, x}^{\alpha, \beta, \eta, \mu} f(x) \Gamma(1-\beta) \Gamma(\alpha+\mu+1+\eta)}{x^{-\mu-\beta} \Gamma(\mu+1) \Gamma(1-\beta+\eta)}\right)\right]^{\frac{q}{p}} d x\right)^{\frac{1}{q}} \\
\leq C\left(\int_{0}^{b} w(t) \Phi(f(t)) d t\right)^{\frac{1}{p}} .
\end{gathered}
$$

If we choose the function $\Phi: \mathbb{R}^{+} \rightarrow \mathbb{R}$ defined by $\Phi(x)=x^{s}, s>1$, then (2.6) reduces to $(2.3)$.

Corollary 2.3. Let $-\infty<q \leq p<0$, and let the assumptions of the Theorem 2.2 be satisfied. If

$$
\begin{gathered}
B=\left(\frac{\Gamma(1-\beta) \Gamma(\alpha+\mu+1+\eta)}{\Gamma(\mu+1) \Gamma(1-\beta+\eta)}\right)^{\frac{1}{p}} \inf _{t \in(0, b)} w^{\frac{-1}{p}}(t) \\
\times\left(\int_{t}^{b} u(x)\left(x^{-\alpha-\mu}{ }_{2} F_{1}\left(\alpha+\beta+\mu,-\eta, \alpha ; 1-\frac{t}{x}\right) t^{\mu}(x-t)^{\alpha-1}\right)^{\frac{q}{p}} d x\right)^{\frac{1}{q}}<\infty,
\end{gathered}
$$


then there exists a positive constant $C$, such that the inequality

$$
\begin{gathered}
\left(\int_{0}^{b} u(x)\left(\frac{I_{0, x}^{\alpha, \beta, \eta, \mu} f(x) \Gamma(1-\beta) \Gamma(\alpha+\mu+1+\eta)}{x^{-\mu-\beta} \Gamma(\mu+1) \Gamma(1-\beta+\eta)}\right)^{\frac{s q}{p}} d x\right)^{\frac{1}{q}} \\
\geq C\left(\int_{0}^{b} w(t) f^{s}(t) d t\right)^{\frac{1}{p}}
\end{gathered}
$$

holds for all measurable functions $f: I \rightarrow \mathbb{R}$ with values in $I$. Moreover, if $C$ is the smallest constant for (2.7) to hold, then $C \geq B$.

Theorem 2.4. Let $1<p \leq q<\infty$, and $\alpha>0, \mu>-1, \beta, \eta \in \mathbb{R}$. Let $u$ be a weight function on $(0, b), w$ be a.e. positive function on $(0, b)$. Suppose that $\Phi: I \rightarrow[0, \infty)$ is a bijective convex function on an interval $I \subseteq \mathbb{R}$. If there exist a real parameter $s \in(1, p)$ and $V:(0, b) \rightarrow \mathbb{R}$ is a positive measurable function such that

$$
\begin{aligned}
& A(s, V)= \\
& \left(\frac{\Gamma(1-\beta) \Gamma(\alpha+\mu+1+\eta)}{\Gamma(\mu+1) \Gamma(1-\beta+\eta)}\right)\left(\int_{0}^{b} V^{\frac{-p^{\prime}(s-1)}{p}}(t) v^{1-p^{\prime}}(t) d t\right)^{\frac{1}{p^{\prime}}} \sup _{t \in(0, b)} V^{\frac{s-1}{p}}(t) \\
& \times\left(\int_{t}^{b} u(x)\left(x_{2}^{-\alpha-\mu} F_{1}\left(\alpha+\beta+\mu,-\eta, \alpha ; 1-\frac{t}{x}\right) t^{\mu}(x-t)^{\alpha-1}\right)^{q} d x\right)^{\frac{1}{q}}<\infty,
\end{aligned}
$$

then there exists a positive constant $C$, such that the inequality

$$
\begin{gathered}
\left(\int_{0}^{b} u(x)\left[\Phi\left(\frac{I_{0, x}^{\alpha, \beta, \mu} f(x) \Gamma(1-\beta) \Gamma(\alpha+\mu+1+\eta)}{x^{-\mu-\beta} \Gamma(\mu+1) \Gamma(1-\beta+\eta)}\right)\right]^{q} d x\right)^{\frac{1}{q}} \\
\leq C\left(\int_{0}^{b} v(t) \Phi^{p}(f(t)) d t\right)^{\frac{1}{p}} .
\end{gathered}
$$

holds. Moreover, if $C$ is the smallest constant for (2.8) to hold, then

$$
C \leq \inf _{\substack{1<s<p \\ V>0}} A(s, V) .
$$

Proof. Applying Theorem 1.3 with the same techniques as used in Theorem 2.2.

Corollary 2.5. If we take $\mu=0$ in Theorem 2.2, Corollary 2.3, Theorem 2.4, we get the results for Saigo fractional derivative. 
Corollary 2.6. If along $\mu=0$ we take $\beta=-\alpha$ in Theorem 2.2, Corollary 2.3, Theorem 2.4, we get the inequalities for Riemann-Liouville's fractional integral which are given in [8] and [7].

Corollary 2.7. If we take $\beta=0$ and $\mu=0$ in Theorem 2.2, Corollary 2.3, Theorem 2.4, we get the inequalities for Erdélyi-Kober fractional integral operator.

\section{HARDY-TYPE INEQUALITIES FOR FRACTIONAL INTEGRAL OPERATOR WHich CONTAINS GENERALIZED MitTAG-LEFFLER FUnCTIONS}

Now we give the definition of Mittag-Leffler function [14] and fractional integral operator involving generalized Mittag-Leffler function appearing in the kernel [17].

Definition 3.1. Let $\alpha, \beta, \gamma, \delta \in \mathbb{C}, \min \{\mathfrak{R}(\alpha), \mathfrak{R}(\beta), \mathfrak{R}(\gamma), \mathfrak{R}(\delta)\}>0$, $m, k>0$ and $k<\mathfrak{R} \alpha+m$. Then the generalized Mittag-Leffler function defined in [17] is given by

$$
E_{\alpha, \beta, m}^{\gamma, \delta, k}(z)=\sum_{n=0}^{\infty} \frac{(\gamma)_{k n}}{\Gamma(\alpha n+\beta)} \frac{z^{n}}{(\delta)_{m n}},
$$

where $(\gamma)_{n}$ represents the Pochhammer symbol, defined by $(\gamma)_{n}=\gamma(\gamma-1)(\gamma-$ $2) \ldots(\gamma-n+1)$. The function (3.1) represents all the previous generalizations of Mittag-Leffler function by setting

- $m=k=1$, it reduces to $E_{\alpha, \beta}^{\gamma, \delta}(z)=\sum_{n=0}^{\infty} \frac{(\gamma)_{n}}{\Gamma(\alpha n+\beta)} \frac{z^{n}}{(\delta)_{n}}$ defined by Salim [16].

- $\delta=m=1$, it represents $E_{\alpha, \beta}^{\gamma, k}(z)=\sum_{n=0}^{\infty} \frac{(\gamma)_{k n}}{\Gamma(\alpha n+\beta)} \frac{z^{n}}{n !}$ which was introduced by A. K. Shukla and J. C. Prajapati in [18]. In [19] H. M. Srivastava and Z. Tomovski investigated the properties of this function and its existence for a wider set of parameters.

- $\delta=m=k=1$, the operator (3.1) is defined by Prabhakar in [15] and is denoted as: $E_{\alpha, \beta}^{\gamma}(z)=\sum_{n=0}^{\infty} \frac{(\gamma)_{n}}{\Gamma(\alpha n+\beta)} \frac{z^{n}}{n !}$.

- $\gamma=\delta=m=k=1$, it reduces to Wiman's function presented in [21], moreover if $\beta=1$, Mittag-Leffler function $E_{\alpha}(z)$ will be the result.

Definition 3.2. Let $\alpha, \beta, \gamma, \delta \in \mathbb{C}, \min \{\mathfrak{R}(\alpha), \mathfrak{R}(\beta), \mathfrak{R}(\gamma), \mathfrak{R}(\delta)\}>0$, $m, k>0$ and $k<\mathfrak{R} \alpha+m$. For all $f \in L(a, b)$ we introduce an integral operator, which contain a Mittag-Leffler function (3.1) in the kernel

$$
\left(\varepsilon_{\alpha, \beta, m, \omega ; a^{+}}^{\gamma, \delta, k} f\right)(x)=\int_{a}^{x}(x-t)^{\beta-1} E_{\alpha, \beta, m}^{\gamma, \delta, k}\left(\omega(x-t)^{\alpha}\right) f(t) d t,
$$


which contains the generalized Mittag-Leffler function (3.1) in its kernel is investigated and its boundedness is proved under certain conditions.

TheOrem 3.3. Let $0<p \leq q<\infty$, and $\alpha, \beta, \gamma, \delta, m, k$ be as in Definition 3.2. Let $u$ be a weight function on $(a, b)$ and $w$ be a.e. positive function on $(a, b)$. Suppose that the function $x \mapsto u(x)\left(\frac{(x-t)^{\beta-1} E_{\alpha, \beta, m}^{\gamma, \delta, k}\left(\omega(x-t)^{\alpha}\right)}{(x-a)^{\beta} E_{\alpha, \beta+1, m}^{\gamma, \delta, k}\left(\omega(x-a)^{\alpha}\right)}\right)^{\frac{q}{p}} i s$ integrable on $(a, b)$ for each fixed $t \in(a, b)$. Let $\Phi$ be a non-negative convex function on an interval $I \subseteq \mathbb{R}$. If

$$
A=\sup _{t \in(a, b)} w^{\frac{-1}{p}}(t)\left(\int_{t}^{b} u(x)\left(\frac{(x-t)^{\beta-1} E_{\alpha, \beta, m}^{\gamma, \delta, k}\left(\omega(x-t)^{\alpha}\right)}{(x-a)^{\beta} E_{\alpha, \beta+1, m}^{\gamma, \delta, k}\left(\omega(x-a)^{\alpha}\right)}\right)^{\frac{q}{p}} d x\right)^{\frac{1}{q}}<\infty
$$

then there exists a positive constant $C$, such that the inequality

$$
\begin{gathered}
\left(\int_{\Omega_{1}} u(x)\left[\Phi\left(\frac{\left(\varepsilon_{\alpha, \beta, m, \omega, a^{+}}^{\gamma, \delta, k} f\right)(x)}{(x-a)^{\beta} E_{\alpha, \beta+1, m}^{\gamma, \delta, k}\left(\omega(x-a)^{\alpha}\right)}\right)\right]^{\frac{q}{p}} d x\right)^{\frac{1}{q}} \\
\leq C\left(\int_{\Omega_{2}} w(t) \Phi(f(t)) d t\right)^{\frac{1}{p}}
\end{gathered}
$$

holds for all measurable functions $f:(a, b) \rightarrow \mathbb{R}$ with values in I. Moreover, if $C$ is smallest constant for (3.3) to hold, then $C \leq A$.

Proof. Applying Theorem 1.1 with $\Omega_{1}=\Omega_{2}=(a, b), d \mu_{1}(x)=d x$, $d \mu_{2}(t)=d t$, where

$$
\tilde{k}(x, t)= \begin{cases}(x-t)^{\beta-1} E_{\alpha, \beta, m}^{\gamma, \delta, k}\left(\omega(x-t)^{\alpha}\right), & a \leq t \leq x \\ 0, & x<t \leq b\end{cases}
$$

(see Lemma 3.2 in [10]), and

$$
\tilde{K}(x)=\int_{a}^{x}(x-t)^{\beta-1} E_{\alpha, \beta, m}^{\gamma, \delta, k}\left(\omega(x-t)^{\alpha}\right) d t=(x-a)^{\beta} E_{\alpha, \beta+1, p}^{\gamma, \delta, k}\left(\omega(x-a)^{\alpha}\right),
$$

we get inequality (3.3).

Corollary 3.4. Let $-\infty<q \leq p<0$, and let the assumption of Theorem 3.3 be satisfied with a positive convex function $\Phi$. If

$$
B=\inf _{y \in(a, b)} w^{\frac{-1}{p}}(y)\left(\int_{t}^{b} u(x)\left(\frac{(x-t)^{\beta-1} E_{\alpha, \beta, m}^{\gamma, \delta, k}\left(\omega(x-t)^{\alpha}\right)}{(x-a)^{\beta} E_{\alpha, \beta+1, p}^{\gamma, \delta,}\left(\omega(x-a)^{\alpha}\right)}\right)^{\frac{q}{p}} d x\right)^{\frac{1}{q}}<\infty
$$


then there exists a positive real constant $C$, such that the inequality

$$
\begin{gathered}
\left(\int_{a}^{b} u(x)\left[\Phi\left(\frac{\left(\varepsilon_{\alpha, \beta, m, \omega, a^{+}}^{\gamma, \delta, k}\right)(x)}{(x-a)^{\beta} E_{\alpha, \beta+1, p}^{\gamma, \delta, k}\left(\omega(x-a)^{\alpha}\right)}\right)\right]^{\frac{q}{p}} d x\right)^{\frac{1}{q}} \\
\geq C\left(\int_{a}^{b} w(y) \Phi(f(y)) d y\right)^{\frac{1}{p}}
\end{gathered}
$$

holds for all measurable functions $f:(a, b) \rightarrow \mathbb{R}$ with values in $(a, b)$. Moreover, if $C$ is smallest constant for (3.4) to hold, then $C \geq B$.

Theorem 3.5. Let $1<p \leq q<\infty$, and $\alpha, \beta, \gamma, \delta, m, k$ be as in Definition 3.2. Let $u$ be a weight function on $(a, b)$, and $v$ be a measurable positive function on $(a, b)$. Suppose that the function $x \mapsto u(x)\left(\frac{\left(\varepsilon_{\alpha, \beta, m, \omega, a}^{\gamma, \delta, k} f\right)(x)}{(x-a)^{\beta} E_{\alpha, \beta, 1, m}^{\gamma, \delta,}\left(\omega(x-a)^{\alpha}\right)}\right)^{q}$ be integrable on $(a, b)$ for each fixed $t \in(a, b)$. Suppose that $\Phi: I \rightarrow[0, \infty)$ is a bijective convex function on an interval $I \subseteq \mathbb{R}$. If there exist a real parameter $s \in(1, p)$ and a positive measurable function $V:(a, b) \rightarrow \mathbb{R}$ such that

$$
\begin{aligned}
& A(s, V)=\left(\int_{a}^{b} V^{\frac{-p^{\prime}(s-1)}{p}}(t) v^{1-p^{\prime}}(t) d t\right)^{\frac{1}{p^{\prime}}} \\
& \times \sup _{t \in(a, b)} V^{\frac{s-1}{p}}(t)\left(\int_{t}^{b} u(x)\left(\frac{\left(\varepsilon_{\alpha, \beta, m, \omega, a^{+}}^{\gamma, \delta, k}\right)(x)}{(x-a)^{\beta} E_{\alpha, \beta+1, m}^{\gamma, \delta, k}\left(\omega(x-a)^{\alpha}\right)}\right)^{q} d x\right)^{\frac{1}{q}}<\infty,
\end{aligned}
$$

then there is a positive real constant $C$, such that the inequality

$$
\begin{gathered}
\left(\int_{a}^{b} u(x) \Phi^{q}\left(\frac{\left(\varepsilon_{\alpha, \beta, m, \omega, a}^{\gamma, \delta, k}\right)(x)}{(x-a)^{\beta} E_{\alpha, \beta+1, m}^{\gamma, \delta, k}\left(\omega(x-a)^{\alpha}\right)}\right) d x\right)^{\frac{1}{q}} \\
\leq C\left(\int_{a}^{b} v(t) \Phi^{p}(f(t)) d t\right)^{\frac{1}{p}}
\end{gathered}
$$

holds for all measurable functions $f:(a, b) \rightarrow \mathbb{R}$ with values in I. Moreover, if $C$ is smallest constant for (3.5) to hold, then

$$
C \leq \inf _{\substack{1<s<p \\ V>0}} A(s, V) .
$$

Proof. Applying Theorem 1.3 with the same technique as used in Theorem 3.3.

REMARK 3.6. Here some special cases of above discussed results are given. 
- If we take $m=k=1$, in Theorem 3.3, Corollary 3.4 and in Theorem 3.5 , the inequality reduces for $E_{\alpha, \beta}^{\gamma, \delta}(z)=\sum_{n=0}^{\infty} \frac{(\gamma)_{n}}{\Gamma(\alpha n+\beta)} \frac{z^{n}}{(\delta)_{n}}$.

- If we take $\delta=m=1$, in Theorem 3.3, Corollary 3.4 and in Theorem 3.5 the inequality reduces for $E_{\alpha, \beta}^{\gamma, k}(z)=\sum_{n=0}^{\infty} \frac{(\gamma)_{k n}}{\Gamma(\alpha n+\beta)} \frac{z^{n}}{n !}$.

- If we take $\delta=m=k=1$, in Theorem 3.3, Corollary 3.4 and in Theorem 3.5 the inequality reduces for $E_{\alpha, \beta}^{\gamma}(z)=\sum_{n=0}^{\infty} \frac{(\gamma)_{n}}{\Gamma(\alpha n+\beta)} \frac{z^{n}}{n !}$.

- If we take $\gamma=\delta=m=k=1$, in Theorem 3.3, Corollary 3.4 and in Theorem 3.5 the inequality reduces to Wiman's function, moreover if $\beta=1$, Mittag-Leffler function $E_{\alpha}(z)$ will be the result.

\section{HARDY-TYPe INEQUALities FOR HilfER FRACTIONAL DERIVATIVE OPERATOR}

Let $x>0$. By $L^{1}(0, x)$ we denote the space of all Lebesgue integrable functions on the interval $(0, x)$. For any $f \in L^{1}(0, x)$ the Riemann-Liouville fractional integral of $f$ of order $\nu$ is defined by

$$
\left(I_{a_{+}}^{\nu} f\right)(s)=\frac{1}{\Gamma(\alpha)} \int_{a}^{s}(x-t)^{\nu-1} f(t) d t=\left(f * K_{\nu}\right)(s), \quad s \in[0, x] \quad(\nu>0),
$$

where $K_{\nu}(s)=\frac{s^{\nu-1}}{\Gamma(\nu)}$. The integral on the right side of (4.1) exists for almost $s \in[0, x]$ and $I_{a_{+}}^{\nu} f \in L^{1}(0, x)$. The Riemann-Liouville fractional derivative of $f \in L^{1}(0, x)$ of order $\nu$ is defined by

$$
\left(D_{a_{+}}^{\nu} f\right)(s)=\left(\frac{d}{d x}\right)^{n}\left(I_{a_{+}}^{n-\nu} f\right)(x),(\nu>0, n=[\nu+1])
$$

By $C^{m}[0, x]$ we denote the space of all functions on $[0, x]$ which have continuous derivatives up to order $m$, and $A C[0, x]$ is the space of all absolutely continuous functions on $[0, x]$. By $A C^{m}[0, x]$ we denote the space of all functions $f \in C^{m}[0, x]$ with $f^{(m-1)} \in A C[0, x]$. By $L_{\infty}(0, x)$ we denote the space of all measurable functions essentially bounded on $[0, x]$. Let $\mu>0, m=[\mu]+1$ and $f \in A C^{m}[a, b]$. The Caputo derivative of order $\mu>0$ is defined as

$$
\left({ }^{C} D_{a_{+}}^{\mu} f\right)(x)=\left(I_{a_{+}}^{m-\mu} \frac{d^{m}}{d x^{m}} f\right)(x)=\frac{1}{\Gamma(m-\mu)} \int_{a}^{x}(x-s)^{m-\mu-1} \frac{d^{m}}{d x^{m}} f(s) d s
$$

Let us recall the definition of Hilfer fractional derivative presented in [20].

Definition 4.1. Let $f \in L^{1}[a, b], f * K_{(1-\nu)(1-\mu)} \in A C^{1}[a, b]$. The fractional derivative operator $D_{a+}^{\mu, \nu}$ of order $0<\mu<1$ and type $0<\nu \leq 1$ with 
respect to $x \in[a, b]$ is defined by

$$
\left(D_{a+}^{\mu, \nu} f\right)(x):=I_{a+}^{\nu(1-\mu)} \frac{d}{d x}\left(I_{a+}^{(1-\nu)(1-\mu)} f(x)\right),
$$

whenever the right hand side exists. The derivative (4.2) is usually called Hilfer fractional derivative.

The more general integral representation of equation (4.2) given in [7] define as: Let $f \in L^{1}[a, b], f * K_{(1-\nu)(n-\mu)} \in A C^{n}[a, b], n-1<\mu<n, 0<$ $\nu \leq 1, n \in \mathbb{N}$ then the following equation holds true:

$$
\left(D_{a+}^{\mu, \nu} f\right)(x)=\left(I_{a+}^{\nu(n-\mu)} \frac{d^{n}}{d x^{n}}\left(I_{a+}^{(1-\nu)(n-\mu)} f(x)\right)\right) .
$$

Specially for $\nu=0, D_{a+}^{\mu, 0} f=D_{a+}^{\mu} f$ is a Riemann- Liouvile fractional derivative of order $\mu$, and for $\nu=1$ it is a Caputo fractional derivative $D_{a+}^{\mu, 1} f={ }^{C} D_{a+}^{\mu} f$ of order $\mu$. Applying the properties of Riemann-Liouvile integral the relation (4.3) can be rewritten in the form:

$$
\begin{aligned}
\left(D_{a+\nu}^{\mu, \nu}\right)(x) & =\left(I_{a+}^{\nu(n-\mu)}\left(\left(D_{a+}^{n-(1-\nu)(n-\mu)} f\right)(x)\right)\right) \\
& =\frac{1}{\Gamma(\nu(n-\mu))} \int_{a}^{x}(x-t)^{\nu(n-\mu)-1}\left(\left(D_{a+}^{\mu+\nu(n-\mu)} f\right)(t)\right) d t .
\end{aligned}
$$

TheOREM 4.2. Let $0<p \leq q<\infty, s>1$. Let $f \in L^{1}[a, b]$ and the fractional derivative operator $D_{a+}^{\mu, \nu}$ of order $n-1<\mu<n$ and type $0<\nu \leq 1$. Let $u$ be a weight function defined on $(a, b), w$ be a.e. positive function on $(a, b)$. If

$$
A=(\nu(n-\mu))^{\frac{1}{p}} \sup _{t \in(a, b)} w^{\frac{-1}{p}}(t)\left(\int_{t}^{b} u(x)\left(\frac{(x-t)^{\nu(n-\mu)-1}}{(x-a)^{\nu(n-\mu)}}\right)^{\frac{q}{p}} d x\right)^{\frac{1}{q}}<\infty
$$

then there exists a positive constant $C$, such that the inequality

$$
\begin{gathered}
\left(\int_{a}^{b} u(x)\left(\frac{\Gamma(\nu(n-\mu)+1)}{(x-a)^{\nu(n-\mu)}}\left(D_{a+}^{\mu, \nu} f\right)(x)\right)^{\frac{s q}{p}} d x\right)^{\frac{1}{q}} \\
\leq C\left(\int_{a}^{b} w(t)\left(\left(D_{a+}^{\mu+\nu(n-\mu)} f\right)(t)\right)^{s} d t\right)^{\frac{1}{p}}
\end{gathered}
$$

holds. Moreover, if $C$ is the smallest constant for (4.5) to hold, then $C \leq A$. 
Proof. Applying Theorem 1.1 with $\Omega_{1}=\Omega_{2}=(a, b), d \mu_{1}(x)=d x$, $d \mu_{2}(t)=d t$ with

$$
\bar{k}(x, t)= \begin{cases}\frac{(x-t)^{\nu(n-\mu)-1}}{\Gamma(\nu(n-\mu))}, & a \leq t \leq x \\ 0, & x<t \leq b\end{cases}
$$

we get

$$
\bar{K}(x)=\frac{(x-a)^{\nu(n-\mu)}}{\Gamma(\nu(n-\mu)+1)} .
$$

The integral operator $A_{k} f(x)$ takes the form

$$
A_{k} f(x)=\frac{\Gamma(\nu(n-\mu)+1)}{(x-a)^{\nu(n-\mu)}}\left(D_{a+}^{\mu, \nu} f\right)(x) .
$$

By using (4.7) in inequality (1.3) we have

$$
\begin{gathered}
\left(\int_{a}^{b} u(x)\left[\Phi\left(\frac{\Gamma(\nu(n-\mu)+1)}{(x-a)^{\nu(n-\mu)}}\left(D_{a+}^{\mu, \nu} f\right)(x)\right)\right]^{\frac{q}{p}} d x\right)^{\frac{1}{q}} \\
\leq C\left(\int_{a}^{b} w(y) \Phi\left(\left(D_{a+}^{\mu+\nu(n-\mu)} f\right)(t)\right) d y\right)^{\frac{1}{p}}
\end{gathered}
$$

For $s>1$, the function $\Phi: \mathbb{R}^{+} \rightarrow \mathbb{R}$ defined by $\Phi(x)=x^{s}$ reduced (4.8) in (4.5).

Corollary 4.3. Let $-\infty<q \leq p<0$, and let the assumption of Theorem 4.2 be satisfied with a positive convex function $\Phi$. If

$$
B=\inf _{t \in(a, b)} w^{\frac{-1}{p}}(t)\left(\int_{t}^{b} u(x)\left(\frac{\nu(n-\mu)(x-t)^{\nu(n-\mu)-1}}{(x-a)^{\nu(n-\mu)}}\right)^{\frac{q}{p}} d x\right)^{\frac{1}{q}}<\infty
$$

then there exists a positive constant $C$, such that the inequality

$$
\begin{gathered}
\left(\int_{a}^{b} u(x)\left(\frac{\Gamma(\nu(n-\mu)+1)}{(x-a)^{\nu(n-\mu)}}\left(D_{a+}^{\mu, \nu} f\right)(x)\right)^{\frac{s q}{p}} d x\right)^{\frac{1}{q}} \\
\geq C\left(\int_{a}^{b} w(t)\left(\left(D_{a+}^{\mu+\nu(n-\mu)} f\right)(t)\right)^{s} d t\right)^{\frac{1}{p}}
\end{gathered}
$$

holds. Moreover, if $C$ is the smallest constant for (4.9) to hold, then $C \geq B$. 
Theorem 4.4. Let $1<p \leq q<\infty$. Let $f \in L^{1}[a, b]$ and the fractional derivative operator $D_{a+}^{\mu, \nu}$ of order $n-1<\mu<n$ and type $0<\nu \leq 1, u$ be a weight function defined on $(a, b), w$ be a.e. positive function on $(a, b)$. If there exist a real parameter $s \in(1, p)$ and a positive measurable function $V:(a, b) \rightarrow \mathbb{R}$ such that

$$
\begin{aligned}
A(s, V)=\nu(n-\mu) & \left(\int_{a}^{b} V^{\frac{-p^{\prime}(s-1)}{p}}(t) v^{1-p^{\prime}}(y) d t\right)^{\frac{1}{p^{\prime}}} \\
& \times \sup _{t \in(a, b)} V^{\frac{s-1}{p}}(y)\left(\int_{t}^{b} u(x)\left(\frac{(x-t)^{\nu(n-\mu)-1}}{(x-a)^{\nu(n-\mu)}}\right)^{q} d x\right)^{\frac{1}{q}}<\infty,
\end{aligned}
$$

then there exists a positive constant $C$ such that the inequality

$$
\begin{aligned}
& \left(\int_{a}^{b} u(x)\left(\frac{\Gamma(\nu(n-\mu)+1)}{(x-a)^{\nu(n-\mu)}}\left(D_{a+}^{\mu, \nu} f\right)(x)\right)^{t q} d x\right)^{\frac{1}{q}} \\
& \leq C\left(\int_{a}^{b} v(t)\left(\left(D_{a+}^{\mu+\nu(n-\mu)} f\right)(t)\right)^{t p} d t\right)^{\frac{1}{p}}
\end{aligned}
$$

holds. Moreover, if $C$ is the smallest constant for (4.10) to hold, then

$$
C \leq \inf _{\substack{1<s<p \\ V>0}} A(s, V) .
$$

Proof. Applying Theorem 1.3 with the same technique used in Theorem 4.2 .

REmark 4.5. In particular if $\nu=0$ in Theorem 3.3, Corollary 3.4 and in Theorem 3.5 the inequalities becomes for Riemann-Liouville fractional derivative of order $\mu$ and for $\nu=1$ then results becomes for Caputo fractional derivative of order $\mu$.

\section{ACKNOWLEDGEMENTS.}

The author Josip Pečarić has been supported by Croatian Science Foundation under the project 5435 . We thank the referee for some valuable advice, which have improved the final version of this paper.

\section{REFERENCES}

[1] A. Čižmešija, K. Krulić and J. Pečarić, On a new class of refined discrete Hardy-type inequalities, Banach J. Math. Anal. 4 (2010), 122-145.

[2] A. Čižmešija, K. Krulić and J. Pečarić, Some new refined Hardy-type inequalities with kernels, J. Math. Inequal. 4 (2010), 481-503. 
[3] A. Čižmešija, K. Krulić and J. Pečarić, A new class of general refined Hardy type inequalities with kernels, Rad Hrvat. Akad. Znan. Umjet. Mat. Znan. 17 (2013), 53-80.

[4] L. Curiel and L. Galue, A generalization of the integral operators involving the Gauss hypergeometric function, Rev. Técn. Fac. Ingr. Univ. Zulia 19 (1996), 17-22.

[5] N. Elezović, K. Krulić and J. Pečarić, Bounds for Hardy type differences, Acta Math. Sin. (Engl. Ser.) 27 (2011), 671-684.

[6] I. S. Gradshteyn and I. M. Ryzhik, Table of Integrals, Series, and Products, Seventh Edition, Elsevier Inc., Amsterdam, 2007.

[7] R. Hilfer, Y. Luchko and Ž. Tomovski, Operational method for solution of fractional differential equations with generalized Riemann-Liouville fractional derivative, Fract. Calc. Appl. Anal. 12 (2009), 299-318.

[8] S. Iqbal, K. Krulić Himmelreich and J. Pečarić, A new class of Hardy-type integral inequalities, Math. Balkanica (N. S.) 28, Fasc. 1-2, (2014), 3-16.

[9] S. Iqbal, K. Krulić Himmelreich and J. Pečarić, On a new class of Hardy-type inequalities with fractional integrals and fractional derivatives, Rad Hrvat. Akad. Znan. Umjet. Mat. Znan. 18 (2014) 91-105.

[10] S. Iqbal, J. Pečarić, M. Samraiz and Ž. Tomovski, Hardy-type inequalities for generalized fractional integral operators, Tbil. Math. J. 10 (2017), 75-90.

[11] S. Kaijser, L. Nikolova, L.-E. Persson and A. Wedestig, Hardy type inequalities via convexity, Math. Inequal. Appl. 8 (2005), 403-417.

[12] K. Krulić, J. Pečarić,and L.-E. Persson, Some new Hardy-type inequalities with general kernels, Math. Inequal. Appl. 12 (2009), 473-485.

[13] K. Krulić, J. Pečarić and D. Pokaz, Inequalities of Hardy and Jensen, Zagreb, Element, 2013.

[14] G. M. Mittag-Leffler, Sur la nouvelle fonction, C. R. Acad. Sci. Paris. 137 (1903), 554-558.

[15] T. R. Prabhakar, A singular integral equation with a generalized Mittag-Leffler function in the kernel, Yokohama Math. J. 19 (1971), 7-15.

[16] T. O. Salim, Some properties relating to the generalized Mittag-Leffler function, Adv. Appl. Math. Anal. 4 (2009), 21-30.

[17] T. O. Salim and A. W. Faraj, A generalization of Mittag-Leffler function and integral operator associated with fractional calculus, J. Fract. Calc. Appl. 5 (2012), 1-13.

[18] A. K. Shukla and J. C. Prajapati, On a generalization of Mittag-Leffler function and its properties, J. Math. Anal. Appl. 336 (2007), 797-811.

[19] H. M. Srivastava and Z. Tomovski, Fractional calculus with an integral operator containing generalized Mittag-Leffler function in the kernal, Appl. Math. Comput. 211 (2009), 198-210.

[20] Ž. Tomovski, R. Hilfer and H. M. Srivastava, Fractional and Operational Calculus with Generalized Fractional Derivative Operators and Mittag-Leffler Type Functions, Integral Transforms Spec. Funct. 21 (2010), 797-814.

[21] A. Wiman, Über den fundamentalsatz in der theorie der functionen, Acta Math. 29 (1905), 191-201. 
Težinske nejednakosti Hardijevog tipe pomoću frakcijskog računa operatora

Sajid Iqbal, Josip Pečarić, Muhammad Samraiz i Živorad Tomovski

SAŽETAK. Cilj ovog rada je izložiti novu generaliziranu klasu težinskih nejednakosti Hardijevog tipa, za klasu konveksnih funkcija, s primjenom generaliziranog frakcijskog računa konvolutivnih integralnih operatora, koje u svojoj jezgri sadrže Gaussovu hipergeometrijsku funkciju, generaliziranu funkciju Mittag-Lefflera, te Hilferov frakcijski diferencijalni operator.

Sajid Iqbal

Department of Mathematics

University of Sargodha, Sub-Campus Mianwali

Mianwali, Pakistan

E-mail: sajid_uos2000@yahoo.com, dr.sajid@uos.edu.pk

Josip Pečarić

Faculty of Textile Technology

University of Zagreb

Zagreb, Croatia

E-mail: pecaric@element.hr

Muhammad Samraiz

Department of Mathematics

University of Sargodha

Sargodha, Pakistan

E-mail: msamraiz@uos.edu.pk

Živorad Tomovski

Faculty of Mathematics and Natural Sciences

Gazi Baba bb

1000 Skopje, Macedonia

E-mail: tomovski@pmf.ukim.mk

Received: 15.5.2017.

Revised: 13.6.2017. 